\title{
LAS ISLAS DEL PACÍFICO
}

Carlos Mondragón

El Colegio de México

El Océano Pacífico ocupa más de 180 millones de kilómetros cuadrados, un área equivalente a la mitad de la superficie marítima de la Tierra. Dispersas sobre esta inmensa extensión de agua se encuentran más de 25000 islas, de las cuales 200 son grandes y 2500 pequeñas, todas habitadas de manera permanente desde hace más de tres milenios. La geografía humana de este continente oceánico se concentra en torno a una veintena de naciones, Estados y territorios conocidos como las Islas del Pacífico. Excluyendo a Nueva Zelanda, la población total de las Islas del Pacífico asciende a 8.5 millones de personas - una cifra que se eleva a más de 15 millones si se incluyen Nueva Zelanda y el archipiélago de Hawai- Este horizonte humano está integrado por más de 1200 grupos lingüísticos, lo cual hace del Oceáno Pacífico la región cultural de mayor extensión y diversidad lingüística del mundo. ${ }^{1}$

El término Asia-Pacífico suele concentrar nuestra mirada sobre las grandes naciones pertenecientes a la cuenca del Pacífico, ubicadas sobre los litorales y la zona oriental del continente asiático. Pero los datos anteriores nos advierten que resulta incompleta una visión geopolítica de aquella macro-región del mundo que no atienda al enorme valor estratégico, de riqueza cultural, de recursos primarios, de nexos políticos internacionales, de intereses comerciales y rutas marítimas, de una arquitectura diplomática y regional singular, así como de la diversidad física y climática que concentran las Islas del Pacífico.

El presente artículo marca la primera ocasión en que se incluyó a las Islas del Pacífico en el Anuario Asia-Pacífico del Centro de Estudios de Asia y África. Con ello se resuelve la

${ }^{1}$ Conviene advertir que el término Islas del Pacífico no incluye a Australia, pero que en conjunto Australia y las Islas del Pacífico constituyen el continente de Oceanía. 
necesaria representación de la totalidad de Oceanía, el continente más extenso de AsiaPacífico y del mundo, constituido por Australia y Nueva Zelanda, junto con las 22 naciones miembro de la Comunidad del Pacífico (cuadro 4 y mapa 1), así como la veintena de Estados insulares y territorios dependientes de países miembros de la APEC (véase el apéndice).

En el transcurso del último año, los más de veinte países que conforman a las Islas del Pacífico han ocupado un sitio prominente en los temas de actualidad de Asia-Pacífico y del mundo. Tres ejemplos importantes han incluido: el liderazgo que demostró Fiji como país encargado de la presidencia de la Asamblea General de Naciones Unidas para el periodo 2016-2017 en materia de política ambiental y legal en relación con la gestión de los océanos y mares del mundo; la celebración de un referéndum, en octubre de 2018, para determinar la independencia o continuidad de la nación de Kanaky/Nueva Caledonia en su relación de territorio dependiente de Francia en el Pacífico; el reconocimiento de ascendencia política y económica de Papúa Nueva Guinea al ser elegido como país anfitrión para la celebración del Foro para la Cooperación Económica de Asia-Pacífico (APEC, por sus siglas en inglés) en noviembre de 2018.

La síntesis de temas de actualidad que se ofrece en las siguientes páginas se ha organizado en torno a un panorama general del perfil socioeconómico de las Islas del Pacífico, seguido de tres ejes determinantes de la actividad social, diplomática, comercial y política que dan forma al Pacífico contemporáneo. Estos ejes son:

1) El desarrollo, entendido desde la perspectiva del desarrollo humano pero incluyente del desarrollo de instituciones, organismos y prácticas de gobierno que responden a los retos y oportunidades de un continente marítimo caracterizado por países insulares pequeños en relación con grandes potencias dominantes.

2) La seguridad, entendida por medio de los dos modelos contrapuestos de seguridad regional que dan forma a la actual arquitectura militar, estratégica y política del Océano Pacífico. Como se verá, dentro de uno de estos dos modelos se incluye de manera prominente el concepto de seguridad humana en el contexto de retos medioambientales y de desarrollo social.

3) El cambio climático y la política ambiental como retos emergentes que desde el inicio del siglo XXI han ido cobrando una urgencia y predominancia determinantes para la 
política social y ambiental en relación con la calidad de vida, y en algunos casos la existencia misma, de algunas de las comunidades humanas que conforman las naciones isleñas de esta región.

En vista de que Australia y Nueva Zelanda cuentan con sus textos respectivos en este anuario, el presente artículo se concentra de manera preferencial sobre las Islas, aunque necesariamente incluye también comentarios acerca de las relaciones entre las Islas del Pacífico, Australia y Nueva Zelanda. La lista de los países que comprenden las Islas del Pacífico se basa en los criterios y límites políticos formales de la Comunidad del Pacífico (CP) y el Foro de Islas del Pacífico (FIP), las dos principales agrupaciones regionales en torno a las cuales se organizan los países y territorios insulares del Oceáno Pacífico. Estos criterios y límites no coinciden completamente debido a la naturaleza misma de ambas organizaciones - la CP es una agrupación dedicada al desarrollo regional, mientras que el FIP es un foro regional que reúne a jefes de Estado y gobierno para asuntos diplomáticos y políticos- Con esto en mente, los pormenores de ambos organismos se explican a continuación (Pacific Community, 2015; Pacific Islands Forum, 2018). 


\section{CuADRO 1. Países independientes (Oceanía)}

\begin{tabular}{|c|c|c|c|}
\hline Países independientes & $\begin{array}{c}\text { Población } \\
\text { (estimada en 2016) }\end{array}$ & Capital & Idiomas oficiales \\
\hline Australia & 20172332 & Canberra & Inglés \\
\hline Fiji & 915303 & Suva & Inglés, fijiano, hindi \\
\hline Kiribati & 106925 & Tarawa & Inglés, gilbertés \\
\hline Islas Marshall & 73376 & Majuro & Marshalés, inglés \\
\hline Islas Salomón & 635027 & Honiara & $\begin{array}{l}\text { Solomons pidgin, } \\
\text { inglés }\end{array}$ \\
\hline $\begin{array}{c}\text { Estados Federados de } \\
\text { Micronesia }\end{array}$ & 104719 & Palikir & Inglés \\
\hline Nauru & 9591 & Yaren & Nauruano, inglés \\
\hline Nueva Zelanda & 4474549 & Wellington & Inglés, maorí \\
\hline Palau & 21347 & Ngerulmud & $\begin{array}{l}\text { Palauano, inglés, } \\
\text { japonés, angaur, } \\
\text { tobiano y sonsorol }\end{array}$ \\
\hline Papúa Nueva Guinea & 6791317 & Port Moresby & $\begin{array}{l}\text { Tok pisin, inglés, hiri } \\
\text { motu }\end{array}$ \\
\hline Samoa & 198926 & Apia & Samoano, inglés \\
\hline Tonga & 106513 & Nuku'alofa & Tongano, inglés \\
\hline Tuvalu & 10959 & Funafuti & Tuvaluano, inglés \\
\hline Vanuatu & 277554 & Port Vila & $\begin{array}{l}\text { Bislama, inglés, } \\
\text { francés }\end{array}$ \\
\hline
\end{tabular}


CUADRO 2. Territorios dependientes (Oceanía)

\begin{tabular}{|c|c|c|c|}
\hline Nombre & Población & Capital & País dominante \\
\hline Guam & 162742 & Agaña & Estados Unidos \\
\hline $\begin{array}{l}\text { Islas Ashmore y } \\
\text { Cartier }\end{array}$ & - & - & Australia \\
\hline Islas Chatham & 717 & & Nueva Zelanda \\
\hline Atolón Johnson & & & Estados Unidos \\
\hline Islas Midway & & & Estados Unidos \\
\hline Atolón Palmyra & & & Estados Unidos \\
\hline Isla Wake & & & Estados Unidos \\
\hline Arrecife Kingman & & & Estados Unidos \\
\hline Isla Jarvis & & & Estados Unidos \\
\hline Isla Baker & & & Estados Unidos \\
\hline Isla Howland & & & Estados Unidos \\
\hline Islas Cook & 9556 & Avarua & Nueva Zelanda \\
\hline $\begin{array}{l}\text { Islas del Mar del } \\
\text { Coral }\end{array}$ & & & Australia \\
\hline $\begin{array}{c}\text { Islas Marianas del } \\
\text { Norte }\end{array}$ & 53467 & Saipan & Estados Unidos \\
\hline Islas Pitcairn & 54 & Adamstown & Reino Unido \\
\hline $\begin{array}{c}\text { Islas de Ultramar } \\
\text { Menores }\end{array}$ & & & Estados Unidos \\
\hline Niue & 1190 & Alofi & Nueva Zelanda \\
\hline Isla Norfolk & 2210 & Kingston & Australia \\
\hline $\begin{array}{l}\text { Nueva Caledonia } \\
\text { (Kanaky) }\end{array}$ & 275355 & Nouméa & Francia \\
\hline Polinesia Francesa & 285321 & Pape'ete & Francia \\
\hline Samoa Americana & 54194 & Pago Pago & Estados Unidos \\
\hline Tokelau & 1337 & & Nueva Zelanda \\
\hline Wallis y Futuna & 15664 & Mata-Utu & Francia \\
\hline
\end{tabular}


CUADRO 3. Territorios oceánicos integrados en estados no-oceánicos

\begin{tabular}{|c|c|c|c|}
\hline Nombre & Población & Capital & País dominante \\
\hline Hawai & 1431603 & Honolulu & Estados Unidos \\
\hline $\begin{array}{c}\text { Rapanui } \\
\text { (Isla de Pascua) }\end{array}$ & 5761 & Hanga Roa & Chile \\
\hline Maluku & 1895000 & Ambon & Indonesia \\
\hline Papúa & 3486432 & Jayapura & Indonesia \\
\hline Papúa Occidental & 60855 & Manokwari & Indonesia \\
\hline
\end{tabular}

\section{PERFIL POLÍTICO Y ECONÓMICO DEL PACÍFICO INSULAR}

La Comunidad del Pacífico, hasta 2015 conocida como Secretaría de la Comunidad del Pacífico (SPC), es la principal organización intergubernamental dedicada a la actividad científica y técnica para el desarrollo en el Pacífico. Se define como una organización gobernada por 26 países y territorios dependientes de naciones exteriores, que tiene el objetivo de "desarrollar las capacidades técnicas, profesionales, científicas, investigativas, de planeación y de manejo de los pueblos de las islas del Pacífico [para] proveer directamente información y asesoría que permitan tomar decisiones informadas sobre su desarrollo futuro y bienestar" (Pacific Community, 2015: 1). La sede de la CP se encuentra en Nouméa, la capital del territorio francés de Kanaky/Nueva Caledonia, aunque tiene oficinas y sedes de secretarías regionales en varias capitales isleñas.

Por su parte, el Foro de las Islas del Pacífico es una organización diplomática intergubernamental que todos los años reúne a los líderes de 18 países pertenecientes a Oceanía. El país miembro más pequeño es Niue, con una población de 1600 personas, mientras que el más grande es Australia, con más de 25 millones de personas. (Conviene reiterar que Australia no forma parte de las Islas del Pacífico, mientras que Nueva Zelanda sí se considera parte de esta región, por razones históricas y culturales.) Establecido en 1971, el FIP fue conformado "para trabajar en apoyo de los gobiernos miembro, para mejorar el bienestar económico y social de la gente del Pacífico Sur” (Moon, 2018). Aunque su misión 
se equipara con la de la Comunidad del Pacífico, el FIP tiene un carácter más diplomático, en el que sus presidentes, primeros ministros y ministerios de asuntos exteriores suelen dar prioridad a sus órganos consultivos. El FIP también atrae a diversos países observadores relevantes, entre ellos la Organización de las Naciones Unidas, el Banco Mundial y los llamados 'socios de diálogo', que incluyen a Indonesia, Alemania, Japón, China, India, Estados Unidos, Reino Unido, y varios más.

El crecimiento económico en el Pacífico ha sido históricamente limitado debido, primero, al relativo estancamiento en el que tuvieron que sobrellevar diversas experiencias de explotación colonial e imperial durante los siglos XIX y XXI, y posteriormente, debido a las débiles estructuras de gobierno y desarrollo económico que heredaron de los procesos de independencia. Así por ejemplo, cuatro de las naciones pertenecientes a esta región siguen apareciendo en la lista de los Países Menos Desarrollados que genera cada tres años la Organización de las Naciones Unidas (estos países son Tuvalu, Islas Salomón, Vanuatu y Kiribati). Sin embargo, desde 2005 ha habido una modesta tendencia a la alza en el PIB de muchos de los países insulares que apunta hacia horizontes prometedores de desarrollo económico y social, aunado al relativo bienestar de las mayorías poblacionales de diferentes archipiélagos (Crocombe, 2001).

Las industrias clave que apuntalan estos nuevos horizontes de desarrollo en las Islas del Pacífico incluyen los sectores asalariados pertenecientes al servicio público, el turismo, los servicios financieros, las cuotas para emisión de licencias para pesca en aguas de las Zonas Económicas Exclusivas de la CP, las remesas que supone la migración estacional en Australia y Nueva Zelanda, y la agricultura doméstica y para mercados de exportación.

Sin embargo, conviene entender la distribución de estas actividades, pues tan importantes resultan las tendencias de mercados formales y las cifras generales de crecimiento económico basadas en sectores productivos, como el enorme valor agregado que suponen los altos niveles de autosuficiencia que generan los esfuerzos cotidianos de una proporción importante de la población de las Islas.

Por ejemplo, mientras que el turismo representa casi la mitad del PIB de países como Palau e Islas Cook, representa únicamente una cuarta parte del de Vanuatu, y menos de una quinta parte del que tiene el gran archipiélago de Kiribati —en donde la mayor parte del ingreso del gobierno proviene de la emisión de licencias de pesca a naciones no-oceánicas-. 
Sin embargo, en países insulares grandes, como Islas Salomón, Vanuatu, Fiji, y sobre todo Papúa Nueva Guinea, la modesta proporción de ingresos por turismo se obtiene que contrastar con un sector agrícola que se distingue sobre todo por haber podido abrirse paso poco a poco a la exportación de productos como el café orgánico de excelente calidad, a mercados internacionales.

Sin embargo el verdadero valor del sector agrícola en estas naciones del Pacífico occidental reside en la enorme proporción de sus poblaciones, que mantienen regímenes sumamente robustos de autosuficiencia alimenticia, lo cual se debe a que en la actualidad la mayor parte de la población de las Islas del Pacífico vive en comunidades rurales de pequeña escala, practica algún tipo de agricultura de subsistencia (en algunas islas, suplementada de manera importante con la pesca artesanal) y mantiene diversos canales de conexión con mercados laborales, formales e informales. Esta, autosuficiencia, marcada como abundancia, ha sido denominada "subsistencia plus" por especialistas en desarrollo humano y agropecuario, y se traduce en una sorprendente capacidad de resiliencia y de seguridad alimenticia para incontables familias y comunidades del Pacífico — con cifras superiores a casi cualquier otro escenario regional en Asia o África-. En ausencia de estos formidables excedentes de producción doméstica, muchas comunidades oceánicas se verían sujetas a niveles críticos de pobreza y vulnerabilidad respecto a su capacidad básica para procurarse alimentos.

Para decirlo de otra manera, el hambre casi nunca ha sido un factor producto de vulnerabilidades estructurales al interior de las sociedades oceánicas. Más importante aún, estos niveles de excedentes en la producción doméstica son resultado directo de estructuras sociales y culturales milenarias, arraigadas en torno a la agricultura trashumante y a formas sumamente robustas de identidad territorial. Ésta es una de las diversas esferas del bienestar y desarrollo humanos del Pacífico en la que puede ponerse de relieve el enorme capital que supone la herencia y continuidad cultural en la región.

En contraste con las naciones agrícolas de Melanesia, muchas de las economías más pequeñas de Polinesia y Micronesia dependen de manera desproporcionada del ingreso que les suponen la ayuda internacional y las remesas de sus connacionales residentes en las naciones prósperas de la cuenca del Pacífico, especialmente Australia, Nueva Zelanda, y en algunos casos los Estados Unidos. La lista de naciones que se encuentran en esta condición 
incluye sobre todo a los Estados Federados de Micronesia, Kiribati, Islas Marshall, Nauru, Niue y Tuvalu, para quienes la ayuda internacional representa poco más de una tercera parte del Producto Interno Bruto. Más recientemente, Vanuatu, Islas Salomón, Fiji y Papúa Nueva Guinea (las islas pertenecientes a Melanesia, que junto con Polinesia y Micronesia conforma las divisiones culturales de Oceanía) han comenzado a gozar de un aumento considerable de remesas producto de programas de jornaleros estacionales establecidos en Australia y Nueva Zelanda. Estos flujos de riqueza han comenzado a tener un impacto considerable en el poder adquisitivo, y en la capacidad de pago por servicios básicos como educación, salud y transporte, de incontables aldeas y familias rurales en Melanesia, y están contribuyendo a la posibilidad de un desarrollo aumentado y estable de estos países en el futuro cercano y medio.

Las remesas también son importantes para naciones isleñas de Polinesia que mantienen estrechos lazos históricos de migración y movilidad con Australia y Nueva Zelanda, éste es el caso de Tonga, Samoa, Islas Cook y Niue. En conjunto, estos lazos y la codependencia migratoria y laboral suponen un factor clave para la viabilidad y el futuro de las poblaciones isleñas de Oceanía con miras al peligro que varias de ellas enfrentan como resultado del cambio climático. En breve, la necesidad que supondrá una migración forzada por el calentamiento global y el alza de niveles del mar se verá parcialmente mitigada por la existencia de una diáspora histórica, diversa, integrada y laboralmente estable en las naciones más grandes que circundan a la cuenca del Pacífico.

En contraste con el panorama de abundancia rural y de flujos monetarios por remesas, un reto que ha ido creciendo a lo largo del último medio siglo para las Islas del Pacífico se deriva del notable incremento en el crecimiento urbano y la migración rural-urbana, debido al incremento de flujos de personas, servicios y mercancías entre mercados y regiones internas: en muchas naciones isleñas estos incrementos se centran en el crecimiento del sector turismo; en el incremento de inversión en proyectos de infraestructura, con su acompañante demanda de mano de obra no calificada, y en el incremento en los niveles de educación y de mercados laborales que se aglutinan en capitales nacionales. Asimismo, se debe al fortalecimiento y en muchos casos la expansión de los cuadros burocráticos de gobierno en diversos países isleños, lo cual resulta crucial toda vez que los trabajadores del Estado suelen representar los sectores asalariados más grandes e influyentes en las economías de mercado, de consumo, y de crecimiento inmobiliario de las naciones isleñas. 
Atendiendo a los enormes contrastes que arrojan las numerosas economías nacionales del Pacífico, se puede observar que los dos países independientes más grandes de la región son Papúa Nueva Guinea, con 8 millones de habitantes y un Producto Interno Bruto de 26 mil millones de dólares, y Fiji, con casi 900000 residentes dispersos sobre un archipiélago de 300 islas y un PIB de 5 mil millones de dólares (Pacific Community, 2015). Dado su tamaño (ocupa la mitad oriental de la segunda isla más grande del mundo después de Groenlandia), el caso de Papúa Nueva Guinea resulta excepcional en relación con cualquier otro país del Pacífico: su economía está dominada por la agricultura y la pesca, pero sobre todo por un sector extractivista que incluye la explotación forestal a gran escala, la extracción primaria de energéticos, especialmente el gas natural, y diversos minerales, de lo cual se deriva que sea sede de algunas de las minas de oro más grandes del mundo. Es este escenario de recursos primarios el que explica el altísimo PIB de Nueva Guinea en comparación con el resto de las Islas del Pacífico, así como el atractivo que sigue suponiendo éste para algunas de las corporaciones extractivistas con presencia global.

En el otro extremo del espectro demográfico y económico se encuentran algunas de las cadenas de islas pequeñas más dilatadas del Pacífico, como Tuvalu y Nauru. Con poco más de 10000 habitantes cada una, las bajísimas tazas poblacionales de estas naciones se contrastan con su extensión geográfica: tomando a la República de Kiribati como ejemplo, los 33 atolones y la Zona Económica Exclusiva de este país insular ocupan 3.5 millones de kilómetros del Océano Pacífico central, una superficie mayor que la de India, pero con tan sólo 120000 habitantes. Las dimensiones y el potencial económicos de estos archipiélagos son necesariamente limitados y por eso se cuentan entre las naciones oceánicas más dependientes de la ayuda internacional. 
CUADRO 4. Población y cifras básicas del PIB e ingreso per cápita de los estados miembros, asociados y observadores de la comunidad del Pacífico

\begin{tabular}{|c|c|c|c|}
\hline Nombre & Población & $\begin{array}{l}\text { Producto Interno } \\
\text { Bruto (en dólares) }\end{array}$ & $\begin{array}{l}\text { Ingreso per Cápita } \\
\text { (en dólares) }\end{array}$ \\
\hline Australia $^{\mathrm{m}}$ & 24125848 & 1388 trillones & 55707 \\
\hline $\begin{array}{c}\text { Estados Federados de } \\
\text { Micronesia }\end{array}$ & 104937 & 329 millones & 3800 \\
\hline $\mathrm{Fiji}^{\mathrm{m}}$ & 884887 & 4869 millones & 5411 \\
\hline Guam $^{\circ}$ & 162742 & 4880 millones & 30500 \\
\hline Islas Cook ${ }^{\mathrm{m}}$ & 17459 & 311 millones & 15002 \\
\hline $\begin{array}{l}\text { Islas Marianas del } \\
\text { Norte }\end{array}$ & 53833 & 682 millones & 13300 \\
\hline Islas Marshall $^{\mathrm{m}}$ & 53158 & 115 millones & 2900 \\
\hline Islas Salomón & 599419 & 1725 millones & 3191 \\
\hline Kiribati $^{\mathrm{m}}$ & 110136 & 167 millones & 1592 \\
\hline Nauru $^{\mathrm{m}}$ & 11200 & 114 millones & 12052 \\
\hline $\begin{array}{l}\text { Nueva Caledonia } \\
(\text { Kanaky })^{\mathrm{m}}\end{array}$ & 278500 & 9890 millones & 38921 \\
\hline Nueva Zelanda $^{\mathrm{m}}$ & 4915480 & 199000 millones & 40266 \\
\hline Niue $^{\mathrm{m}}$ & 1624 & 10 millones & 5800 \\
\hline $\mathrm{Palau}^{\mathrm{m}}$ & 21503 & 300 millones & 16296 \\
\hline Papúa Nueva Guinea $^{\mathrm{m}}$ & 8084999 & 26000 millones & 3123 \\
\hline Samoa $^{\mathrm{m}}$ & 195843 & 1188 millones & 5962 \\
\hline Samoa Americana ${ }^{a}$ & 55689 & 711 millones & 13000 \\
\hline Timor-Leste $^{\mathrm{a}}$ & 1167242 & 4567 millones & 5479 \\
\hline Tonga $^{\mathrm{m}}$ & 100651 & 763 millones & 7344 \\
\hline Tokelau $^{\mathrm{a}}$ & 1499 & 10 millones & 6275 \\
\hline Tuvalu & 11192 & 39 millones & 3566 \\
\hline Vanuatu $^{\mathrm{m}}$ & 272459 & 773 millones & 2631 \\
\hline Walllis y Futuna ${ }^{\circ}$ & 11899 & 188 millones & 12640 \\
\hline
\end{tabular}

NotAs: $m=$ miembros.

$\mathrm{a}=$ asociados.

$\mathrm{o}=$ observadores. 


\section{DESARROLLO REGIONAL}

El marco del actual escenario de desarrollo humano en el Pacífico está estrechamente relacionado con la visión que las potencias dominantes mantienen de la región, sobre todo en relación con su diplomacia y con su idea de responsabilidad y exclusividad en el desarrollo de la región. En ese sentido, las relaciones entre Australia, Estados Unidos, la Unión Europea y otras potencias en el Pacífico dan lugar a un escenario en el que el desarrollo y la idea de seguridad regional son elementos mutuamente constitutivos. En este subapartado se atenderá especialmente el tema del desarrollo, y en el siguiente el de la seguridad, pero sin perder de vista que ambos emergen del mismo tipo de idea que acerca de la región mantienen, por un lado, las potencias externas, y por otro, las naciones del Pacífico.

Los dos países punteros en financiamiento para el desarrollo en el Pacífico siguen siendo Australia y Nueva Zelanda, seguidos de la Unión Europea, Japón y los Estados Unidos. Asimismo, mantienen una presencia e influencia considerables el Banco Asiático de Desarrollo y el Banco Mundial en la ayuda y financiamiento para el desarrollo en la región. Tratándose de Australia y Nueva Zelanda, ambas naciones oceánicas mantienen una fuerte presencia diplomática, comercial, de desarrollo y de cooperación en las Islas del Pacífico. A lo largo de las dos últimas décadas, sin embargo, Australia ha sufrido vaivenes en relación con sus vecinos insulares; esto se debe en gran medida al surgimiento de gobiernos conservadores australianos que han tenido una actitud menos generosa hacia las Islas, al mismo tiempo que han tendido a mirar al Pacífico como un escenario dominado por retos de tipo estratégico y de (des)orden regional (White, 2010; Dornan, 2018).

Parte de esta visión negativa se debe a la propia crisis de credibilidad, al retroceso en el estado de derecho, y sobre todo a la corrupción que en diferente medida se manifiesta en algunos gobiernos isleños —entre los que actualmente ocupan lugares prominentes Papúa Nueva Guinea, Nauru, y en menor medida Fiji, Islas Salomón y Vanuatu-. La corrupción al interior del servicio público es, en efecto, un enorme reto para estos países pequeños, sin embargo, no es homogénea y con frecuencia se ha convertido en el blanco de movimientos sociales y de organizaciones regionales. Otra parte de la visión australiana de una región de inestabilidad se deriva de las crisis que se suscitaron entre 1998 y 2003 en naciones vecinas, como Timor Leste, las Islas Salomón, y en menor medida Papúa Nueva Guinea. 
En el caso de Timor y Salomón, Australia se colocó a la cabeza de sendas intervenciones militares que fueron punta de lanza para una posterior entrada de enormes cantidades de recursos humanitarios y de reconstrucción nacional. En el caso de Papúa Nueva Guinea, así como de Vanuatu, Australia ha mantenido programas de adiestramiento y consolidación de fuerzas policiacas nacionales a partir de la colocación de cantidades importantes de asesores pertenecientes a la Policía Federal australiana. En todos los casos, las misiones de estabilización y reconstrucción tuvo éxitos y fracasos que han dado lugar a un cierto desgaste en la imagen pública de Australia en el Pacífico, al mismo tiempo que generaron una noción doméstica de Melanesia como un "arco de inestabilidad". Estos procesos han resultado en un reposicionamiento regional en el que la política doméstica ha tendido a generar un sentido de "desinterés benigno" por parte de Australia hacia el Pacífico (Dornan, 2018).

Es este reposicionamiento australiano el que ha ofrecido una oportunidad para que tanto China como Nueva Zelanda tracen sus propios mapas de ruta en relación con las Islas del Pacífico. En ambos casos, estas dos naciones han buscado intensificar y mejorar la calidad de su presencia e intervenciones en el desarrollo regional. En el caso de Nueva Zelanda, ha sido clave la actividad propiciada por el gobierno progresista de la primer ministro Jacinda Ardern, quien ha ampliado y mejorado la calidad de los programas de migración y trabajo estacional dirigidos a isleños del Pacífico.

Como se puede apreciar, los procesos y desplazamientos diplomáticos y de desarrollo que marcan actualmente el estado de las relaciones interregionales y extraregionales en el Pacífico no ocurren en un vacío: se desenvuelven de la mano con percepciones estratégicas y de seguridad propias de las potencias dominantes en la cuenca del Pacífico. La situación actual de las políticas de intervención para el desarrollo en las Islas no puede por tanto reducirse a un problema de retos estrechamente ligados al crecimiento económico o a la estabilidad gubernamental de las Islas.

La visión regional paradigmática (histórica) de las potencias grandes y medias regionales está representada por naciones casi todas externas a la realidad local, política y cultural de las Islas del Pacífico. Esta visión fuereña se basa en la noción de que la región sufre de un déficit crónico de gobernanza y subdesarrollo económicos, y que constituye una zona de extensión de la competencia global. Hoy día, son principalmente los Estados Unidos, 
Australia y China los principales participantes de este modelo de relación y presencia en el Pacífico.

El actual escenario derivado de la actuación de aquellas potencias ha tenido su más reciente iteración a partir de 2011, con el anuncio del entonces presidente Barack Obama de que Estados Unidos buscaría un "pivote", un giro, en su política exterior hacia el este de Asia. En varios de los gobiernos de las Islas del Pacífico este anuncio se interpretó positivamente como parte del interés de la secretaria de Relaciones Exteriores (Hillary Clinton) por reanudar el nivel de actividad multilateral, de inversión y comercio, así como de presencia militar, de apoyo gubernamental y ayuda internacional que Estados Unidos tenía en relación con el Pacífico central y occidental. Esta recepción optimista del mensaje de Obama por parte de las naciones isleñas de debía en gran medida al largo estancamiento en el que habían dejado sus relaciones con el Pacífico previas administraciones estadounidenses. Sin embargo, el "pivote" de la administración Obama era una respuesta a la aspiración por desplazar la mirada y efuerzo estadounidenses del Medio Oriente (Afganistán e Irak) al Este de Asia, en respuesta al reto percibido del ascenso estratégico de China en la región. En la medida en que nuevas crisis internacionales en Siria, Corea del Norte y el Mar del Sur de China absorbieron los recursos y la atención de la política exterior de los Estados Unidos, la Comunidad del Pacífico percibió nuevamente marginal a la visión estratégica del gigante estadounidense.

En contraste, la República Popular China había ido aumentando gradualmente su interés y su presencia estratégica en las Islas del Pacífico. Para entender mejor las enormes dimensiones que en años recientes ha cobrado el posicionamiento estratégico de China en Oceanía, conviene entender el horizonte de muy bajo perfil que guardó anteriormente la RPCh en esta región. En el medio siglo entre la década de 1970 y 2000, la presencia de China en las Islas del Pacífico había sido poco prominente. Ésta se centraba en torno a dos fenómenos: el primero, migratorio e informal, es la existencia de una diáspora cantonesa dilatada, producto de los vaivenes de la historia china de mediados del siglo XX, que ha ido estableciendo modestos enclaves de pequeño y mediano comercio en algunas capitales regionales (Puerto Moresby, Suva, Honiara, Puerto Vila, Nuku'alofa), y el segundo, la competencia de varias décadas entre la RPCh y la República de Taiwán por ganar votos de reconocimiento diplomático en las Naciones Unidas a cambio de diversos paquetes de ayuda, apoyo y trato comercial preferencial con pequeñas naciones insulares (Crocombe, 2007). 
Esta situación comenzó a cambiar durante los últimos años de la presidencia de $\mathrm{Hu}$ Jintao. Aproximadamente de 2008 en adelante, la RPCh comenzó a incrementar su ayuda para el desarrollo en diversas naciones del Pacífico. Este interés de China por las Islas del Pacífico se antoja similar al ascenso de su actividad en África y partes del sur de Asia. Como consecuencia, en años recientes ha levantado las alarmas de algunos sectores políticos y estratégicos en Australia y Nueva Zelanda, que siguen siendo por mucho las naciones dominantes en ayuda internacional en Oceanía. Sin embargo, un cuidadoso desglose de los datos concretos acerca de inversiones y ayudas gubernamentales chinas en el Pacífico a la fecha sugiere dimensiones un poco menos alarmantes de lo que harían pensar los encabezados y declaraciones de algunos sectores de la prensa y política australianas.

La situación actual de tensión entre Australia y China por colocarse como países determinantes de las ayudas, los créditos y los proyectos de desarrollo en el Pacífico se debe, como ya se indicó, principalmente a las recientes tendencias de repliegue regional en la política doméstica australiana. Este proceso tuvo sus inicios durante los últimos años del siglo pasado, cuando comenzó a surgir una serie de gobiernos de tendencia conservadora, arraigados en el Partido Liberal. Fue entonces cuando la economía australiana comenzó a beneficiarse de un periodo extraordinario de crecimiento económico basado en parte en reformas de gobierno y en parte en el notable incremento en la exportación de materia prima para el mercado chino (White, 2007).

Simultáneamente, el Partido Liberal comenzó a poner énfasis en el problema de los migrantes ilegales y en los miedos étnicos de la "Australia blanca" como ejes de su política doméstica. Estos procesos han sido bien documentados, y aquí únicamente conviene advertir que han tenido un efecto gradual pero importante de enfriamiento de las relaciones entre Australia y el Pacífico. Fue entonces cuando se aumentó la percepción del Pacífico como una región de inestabilidad con la cual Australia tenía que tratar en términos de su problemático "patio trasero". La condescendencia explícita de esta visión no tardó en ser criticada desde distintos sectores de la propia diplomacia y la academia australianas, pero siguió siendo dominante cuando menos hasta terminada la década de 2000.

El consenso entre analistas del Pacífico es que a lo largo de la pasada década Australia podría haber hecho más por promover el estado de derecho y condenar la corrupción entre países miembros de la CP. Los gobiernos de Papúa Nueva Guinea y Nauru, en particular, han 
tenido retrocesos importantes en relación con el estado de derecho en los últimos años. La política controversial - condenada por Naciones Unidas - del gobierno australiano de mantener centros extraterritoriales de detención de solicitantes de asilo en Manus (Papúa Nueva Guinea) y Nauru restringió la capacidad de Australia para criticar a los gobiernos corruptos de Peter O'Neill (PNG) y Baron Waqa (Nauru). Esta política deshumanizante y violatoria de tratados internacionales en materia de refugiados y migrantes ha polarizado a la opinión doméstica australiana y se ha convertido en un verdadero dolor de cabeza para sucesivas administraciones conservadoras. Por esa razón, en 2016 la Suprema Corte de Papúa Nueva Guinea declaró ilegal el campamento de Manus, con lo que forzó a Australia a retirarse del mismo en octubre de 2017. El proceso de repliegue de la administración australiana del campamento fue caótico y dio lugar a una nueva ronda de críticas por parte de grupos de derechos humanos, domésticos e internacionales. A la fecha, en contraste, el campamento de detención de solicitantes de asilo en Nauru sigue operando a partir de un subcontratista del gobierno federal australiano. Es en gran medida como resultado de los acomodos necesarios para mantener estos campamentos que Australia se ha mostrado renuente a criticar a gobiernos corruptos en el Pacífico (Newton Cain, 2018).

Esta renuencia de Australia por ocupar una posición de liderazgo en relación con valores progresivos en la región se ha visto agudizada en la última década a causa del vacío político que ha generado el Partido Liberal en cuanto al cambio climático. Mientras el resto de la región y la mayoría de la comunidad internacional se han comprometido a lograr metas para la mitigación del calentamiento global, Australia ha mantenido una posición de repliegue en su política ambiental que obstaculiza su labor como potencia regional y diplomática de frente a la Comunidad del Pacífico. Sobre este problema se ofrecen más datos en la última sección del presente texto.

Ahora bien, fue durante esta última década de repliegue australiano que China comenzó a convertirse en un gigante económico y diplomático, con capacidades novedosas de extensión y penetración comercial y regional en el Pacífico.

Poco a poco, diversas naciones isleñas de la Comunidad del Pacífico, en especial aquéllas que parecían haber recibido un trato más negativo por parte de Australia, no tardaron en abrir sus puertas a la generosidad financiera y desarrollista de la República Popular China. El caso temprano más prominente fue el de Fiji durante el régimen golpista del general Frank 
Bainimarama, que había sido rechazado tanto por Australia y Nueva Zelanda como por la Commonwealth británica. Sin embargo, a partir de 2011 la RPCh comenzó a convertirse en un actor relevante en proyectos de inversión y flujos financieros importantes, en lugares como Tonga, Islas Cook y Vanuatu (Chang 2018).

Ahora bien, China sigue siendo un actor intermedio en el Pacífico en términos cuantitativos. Entre 2011 y 2016, Australia y Nueva Zelanda fueron responsables del 55\% de toda la ayuda otorgada a los países miembros de la Comunidad del Pacífico. En contraste, a pesar de sus constantes declaraciones por aumentar su ayuda oceánica, China terminó contribuyendo únicamente con $8 \%$ de toda la ayuda al Pacífico durante ese periodo. En números concretos, mientras que la RPCh declaró su intención de erogar hasta 5880 millones de dólares en ayuda, la cifra real ha sido mucho menor, mientras que Australia canalizó más de 6720 millones de dólares a la región. Esto explica la enorme diferencia en el porcentaje de ayuda erogada entre Australia y Nueva Zelanda, comparada con la de la RPCh (Chang, 2018).

A su vez, es necesario añadir las anteriores cifras a la inversión oficial directa que ha canalizado China hacia grandes proyectos de infraestructura en distintos países isleños; inversiones que, en su mayoría, han tomado la forma de enormes préstamos directos a los gobiernos nacionales en cuestión. En este sentido, la visión estratégica de la RPCh en el Pacífico es consistente con su estrategia en África y el interior de Asia, a saber, depende de colocarse de manera clave en relación con sectores comerciales, industriales, financieros y de inversión pública de países previamente dependientes de la ayuda y el desarrollo internacional es gestionado desde las capitales del mundo euroamericano.

Parte clave de esta colocación se sustenta en la emisión de líneas de crédito (en efecto, de deuda) con condiciones previas mínimas. Eso suele traducirse en un déficit preocupante de transparencia que detona las alarmas de organizaciones y gobiernos exteriores que están tratando de dar seguimiento a regímenes de buena gobernanza y de rendición de cuentas en el Pacífico. Más aún, las facilidades para emitir crédito no están enteramente libres de condiciones: en efecto, los flujos de capital asociados a estos créditos generalmente están amarrados al requerimiento de que las inversiones resultantes favorezcan a compañías e intereses chinos en la nación receptora. Con frecuencia dichos intereses están dedicados a 
colocar intereses e inversiones chinas en el centro de grandes proyectos de infraestructura pública — proyectos con un alto valor estratégico, además de social.

A lo largo del último decenio esto se ha traducido en una repentina presencia china la relación con la construcción de aeropuertos, muelles de gran calado, vías terrestres principales, y espacios públicos como estadios deportivos y parques comerciales importantes en lugares como Vanuatu, Fiji, Tonga y Papúa Nueva Guinea. Las controversias en torno al grado de penetración de dinero y el control chinos de algunos de estos proyectos no se han hecho esperar, e incluso han sido parte del factor que ha motivado a Australia a renovar su interés y presencia en las Islas (Hayward-Jones, 2018).

Aquí, vale la pena señalar que los procesos con los cuales se materializan los apoyos chinos para el desarrollo en el Pacífico - donde las inversiones exteriores regresan por vía de licitaciones a compañías e intereses domésticos- no se diferencian demasiado de la llamada Boomerang Aid (Ayuda Búmerang) australiana, mediante la cual se da esencialmente el mismo tipo de procedimiento. El monto total de estas inversiones entre 2011 y 2016 fue de 1260 millones de dólares; en contraste, Australia erogó 6580 millones en inversiones adicionales a las ayudas arriba citadas (Chang, 2018).

No obstante, en el transcurso del pasado año las comunidades diplomática y de seguridad internacional de Australia y Estados Unidos siguieron alimentando una narrativa de seguridad regional en Asia-Pacífico que proyecta una creciente ansiedad por el repentino y veloz ascenso de la presencia oficial de China en el seno de las Islas. La manera en que esta narrativa se ha desarrollado, sobre todo desde el Parlamento y el gobierno federal australianos, ha generado el riesgo de crear un debate esencialista unidimensional que se enfoca demasiado en los intereses geoestratégicos de las principales potencias, sin reconocer las complejidades de desarrollo de la región, incluidos los desafíos no convencionales, colectivos, que arroja la complejidad geográfica, política y sociocultural de las Islas del Pacífico. Estos desafíos incluyen: el cambio climático, la debilidad estructural de algunos gobiernos insulares, su respectiva capacidad para patrullar adecuadamente sus mares, recursos y rutas comerciales, la amenaza perenne que supone la corrupción en la estabilidad social y financiera de algunas naciones (como Papúa Nueva Guinea, Islas Salomón, y Nauru) y el constante fenómeno de la mobilidad humana que caracteriza a las sociedades insulares en relación con los países de la Cuenca —en particular Australia, Nueva Zelanda y Estados Unidos. 
Como respuesta a estos retos y ansiedades, hacia el último cuarto de 2018 el gobierno australiano decidió aumentar de manera destacada su presencia diplomática, comercial, militar y de ayuda internacional en el Pacífico. Como paso inicial, en noviembre de 2018 el primer ministro Scott Morrison indicó que "Australia creará un fondo de infraestructura de 2 mil millones de dólares australianos que invertirá en proyectos de telecomunicaciones, energía, transporte, agua" (Financial Express, 2018). Asimismo, se declaró la intención de apartar hasta 1 mil millones de dólares australianos para ofrecer préstamos a la iniciativa privada dedicada a las inversiones productivas en la región. Australia indicó, finalmente, que ampliaría su presencia diplomática en Palau, las Islas Marshall, la Polinesia Francesa, Niue y las Islas Cook (Financial Express, 2018).

Mientras que esta nueva iniciativa pudiera parecer poco controversial, no pasó desapercibido por la prensa y los gobiernos de la Comunidad del Pacífico el hecho de que durante el anuncio de los planes australianos el primer ministro Morrison afirmó que "[El Pacífico] es nuestro terreno. Es nuestra parte del mundo" (Ibid.).

Los comentarios y críticas a la esencia de esta iniciativa, junto con la continuidad de un estilo discursivo visiblemente condescendiente de parte de importantes políticos australianos hacia el Pacífico, no se dejaron esperar. Atendiendo únicamente a las críticas sobre el fondo para infraestructura, la principal de éstas se refiere a dos problemas: 1) que este tipo de inyecciones de dinero suelen distorsionar los mercados locales y regionales dedicados a la infraestructura, y 2) que no se ha dicho nada acerca del hecho de que estos fondos serán distribuidos a manera de préstamos, lo que los convierte en una variante más de las "ayudas" actuales que reciben los gobiernos insulares. A esto se suma también el hecho de que no se ha mencionado nada acerca de procesos de transparencia y criterios de costobeneficio en relación con inversiones y créditos específicos emanados del fondo (Dornan, 13 de julio de 2018).

Mientras que éstas y otras interrogantes habrán de resolverse en la medida en que se desarrollen y maduren los planes de Australia para las Islas del Pacífico, este último proceso ilustra bien algunas de las contradicciones y problemáticas que se han suscitado de manera reiterada en las complicadas relaciones entre la potencia dominante regional y el resto de las naciones y territorios de Oceanía. 


\section{SEGURIDAD}

El sistema regional de gobernanza y diplomacia de las Islas del Pacífico está sustentado en un complejo entramado de naciones isleñas representadas principalmente por la Comunidad del Pacífico y el Foro de las Islas del Pacífico, organismos regionales e internacionales, en especial el Banco Mundial, el Banco Asiático de Desarrollo y la Unión Europea, así como diversas potencias, grandes y medias, que incluyen a Estados Unidos, Australia, Nueva Zelanda, Francia, Japón y, en años recientes, China. En el Océano Pacífico se empalman y coexisten ambos modelos y dan lugar a un importante contraste que guarda lecciones interesantes para el análisis de la seguridad en contextos regionales. En este apartado se desglosan ambos modelos con el fin de problematizar y ampliar los horizontes referentes a la seguridad regional en Asia-Pacífico (Herr, 2015; IISS, 2018).

A. El primer modelo nos refiere al paradigma clásico de seguridad nacional basado en la competencia entre Estados nacionales, la presencia, extensión y capacidad de penetración de fuerzas militares, y la visión del Pacífico en términos de zonas de influencia.

Desde hace casi setenta años ha sido preponderante en los mares e islas de Oceanía la presencia, en términos concretos de dominación comercial y militar, de potencias como Estados Unidos, Australia, Japón, Francia, Corea del Sur y, en años recientes, China, junto con algunos países sudamericanos y del sureste de Asia. La forma que toma esta preponderancia es una herencia del modelo de seguridad nacional que impuso Estados Unidos sobre la región transpacífica al final de la Segunda Guerra Mundial. En breve se sustenta en un sistema asimétrico de tratados y alianzas nacionales entre países y potencias regionales donde Estados Unidos ocupa el papel dominante en las esferas diplomática, comercial y, sobre todo, militar.

La forma concreta que toma este sistema se observa sobre todo en la enorme red de infraestructura diplomática, interpenetración comercial y presencia militar e industrial controlada directamente por los estadounidenses en sitios clave de la cuenca e Islas del Pacífico. Debido a que se trata de un sistema vertical, sus contornos se repiten en escala descendente en relación con Japón, Australia y Corea del Sur, las potencias medias más cercanamente aliadas a Washington - aunque la fuerza y forma de esas alianzas han comenzado a desplazarse en las últimas dos décadas, luego del reposicionamiento 
australiano-, el cual paradójicamente es un repliegue nacionalista acompañado de una renovada proyección militarista regional y la reconfiguración comercial, infraestructural y financiera que está generando China como actor emergente de consideración.

Pero tal vez el rasgo más importante de este modelo de seguridad sustentado en la realpolitik de la posguerra es que margina de manera importante la visión y agencia de los países y territorios representativos de las Islas del Pacífico. Se trata de un modelo de dominación estratégica determinado y financiado desde las capitales de potencias internacionales alejadas de la historia, la realidad cotidiana y las formas colectivas de diplomacia y gobernanza de los pueblos y naciones del Pacífico. Sin embargo, mientras los proyectos de las dos grandes potencias regionales - a saber, China y Estados Unidostrascienden los límites del Oceáno Pacífico, sus actividades en el litoral y en el continente asiáticos están fuertemente determinadas por la arquitectura de seguridad existente en las Islas. Por esa razón, conviene desglosar su estado actual, para después pasar al concepto contrastado de seguridad regional que actualmente sustentan las Islas.

No obstante su creciente preocupación por el ascenso de China en la región, Estados Unidos sigue manteniendo una presencia militar predominante en el Pacífico. Para mejor aquilatar y poner en contexto el tono alarmista acerca de la modernización y crecimiento de la armada y ejército chinos en Asia-Pacífico, vale la pena desglosar en detalle la maquinaria militar estadounidense en Oceanía - la cual ha sido el elemento central de la visión de seguridad regional desde finales de la Segunda Guerra Mundial.

Actualmente Estados Unidos cuenta con dos flotas de su Armada en el Pacífico. La llamada Tercera Flota consiste en cinco grupos de ataque con portaaviones, cada uno poseedor de un complemento de cruceros, destructores y fragatas, así como un total de 30 submarinos y 400 aviones de ataque y apoyo. Estos elementos tienen sus cuarteles permanentes en las costas del Pacífico continental, en California y en el estado de Washington, así como en Pearl Harbor, en el archipiélago hawaiano. El área de actividad designada de la Tercera Flota se concentra en el litoral asiático; es ésta la flota encargada de mantener abiertas las rutas marítimas comerciales y militares de mayor importancia estratégica en los estrechos de Malaca y Taiwán, así como en el Mar de Sur de China. En consecuencia, es la flota que con creciente frecuencia está teniendo roces con elementos marítimos y aéreos de la Armada de China en esa última zona. Por su parte, la Séptima Flota 
de la Armada estadounidense está compuesta por tres grupos de ataque con portaaviones y diez cuerpos de las llamadas Fuerzas de Tarea distribuidas en más de una decena de cuarteles y bases aéreas permanentes ubicadas en decenas de bases aéreas y navales distribuidas en Japón (Misawa, Yokota, Atsugi, Yokosuka, Iwakuni, Sasebo, Kadena y Futenma), Singapur, Filipinas, Guam, Corea del Sur, Hawai y California. El área de actividad de la Séptima Flota incluye el Océano Índico, pero recientemente ha estado desplazando sus actividades hacia la península coreana en respuesta a las políticas externas de Obama y Trump hacia esa región del mundo.

En total ambas flotas cuentan con 250000 efectivos, 2000 aviones y 200 barcos, todos apostados en sitios ubicados sobre la cuenca y las Islas del Pacífico. Sin embargo, la presencia estadounidense no se limita a su Armada. Además de los agrupamientos marítimos arriba citados, Estados Unidos mantiene al Ejército del Pacífico (80 000 soldados y personal de apoyo), así como a la Quinta, Séptima y Vigésimoprimera Fuerzas Aéreas (31 200 efectivos y 334 aviones) y dos fuerzas Expedicionarias del Cuerpo de Marines (84 000 efectivos especializados en operaciones anfibias de avanzada) en el escenario del Oceáno Pacífico. Estos cuerpos del Ejército, Fuerza Aérea y Marines dedicados a mantener predominancia militar en el Pacífico están acuartelados en Hawai.

De acuerdo con cifras públicas más recientes, correspondientes a 2010, el costo de manutención de este enorme aparato militar en activo sobre el escenario de Asia-Pacífico supera los 200 mil millones de dólares anuales. Ésta es una cifra decenas de veces mayor que el PIB anual de todas las naciones y territorios de Oceanía en su conjunto, incluyendo a Nueva Zelanda, la Polinesia francesa, Kanaky/Nueva Caledonia y Papúa Nueva Guinea, pero excluyendo a Australia y a Hawai. Es ésta la realidad militar estadounidense en el terreno y el mar oceánicos sobre la cual es necesario contrastar las declaraciones alarmistas en relación con la amenaza percibida que supone el ascenso de China en el Pacífico.

En contraste, la República Popular China mantiene una presencia armada dividida en tres flotas: la Flota del Mar del Norte, acuartelada en Shandong, la Flota del Mar de Oriente, acuartelada en Zhejiang, y la Flota del Mar del Sur, acuartelada en Guangdong. Cada flota consiste en destructores, fragatas, submarinos y aviones de apoyo. La Armada en su totalidad posee sólo un portaaviones en activo, con un segundo portaaviones próximo a entrar en 
operaciones. El total de efectivos de la Armada de Liberación del Pueblo es de 255000 personas, 512 barcos y unos 700 aviones (IISS, 2018).

En vista de la ambiciosa inversión en la ampliación y modernización de sus fuerzas armadas, la RPCh sin duda posee un aparato bélico marítimo suficiente para mantener en alerta a los Estados Unidos en el entorno del litoral asiático. En especial, ha comenzado a cambiar el equilibrio de fuerzas en relación con Estados Unidos, en zonas como el estrecho de Taiwán, y de manera más limitada, en torno a las islas artificiales que ha estado construyendo con fines de presencia militar en el mar del Sur de China. Aquí conviene añadir que los activos militares estadounidenses fueron objeto de niveles incrementados de inversión, renovación y crecimiento durante las administraciones de los presidentes Obama y Trump por igual. En consecuencia, tendría que haber un descalabro mayor en la inversión estadounidense sobre su arquitectura militar existente, en simultaneidad con un crecimiento y extensión desproporcionados de la Armada china - insostenibles a partir de las perspectivas presupuestales actuales de la $\mathrm{RPCh}$ - para poder contemplar un cambio serio en el status quo actual.

En fin, no solo es problemático pensar en un reto militar chino en términos estrechamente numéricos, sino que supone una equivocada lectura de la visión política y estratégica de mediano y largo plazos que mantiene el régimen de la RPCh en las Islas del Pacífico. Esta estrategia se manifiesta en el esfuerzo de China por estabilizar su posición en relación con las naciones próximas al litoral asiático (Asia del Norte tanto como Sureste de Asia), así como por afirmar una presencia militar permanente en sitios antes limítrofes a su zona de influencia regional. El más prominente de éstos es actualmente el Mar de Sur de China, pero también algunos puertos estratégicos en el Océano Índico y en el Pacífico occidental. $^{2}$

${ }^{2}$ Durante el transcurso de la cumbre APEC celebrada en Puerto Moresby a mediados de noviembre de 2018, el vicepresidente de los Estados Unidos, Mike Pence, y el primer ministro de Australia, Scott Morris, anunciaron planes para "echar para atrás la presencia creciente de China en el Sureste asiático" a partir del establecimiento de una base naval conjunta, administrada por ambas naciones, en la isla de Manus, Papúa Nueva Guinea (Hasham, 2018). El vicepresidente Pence declaró que esta base recibirá una inversión inicial de cinco millones de dólares, y tendría como objetivo ampliar las labores de Estados Unidos y Australia para "proteger la soberanía y derechos marítimos de las Islas del Pacífico" (Fish, 2018). Esta iniciativa reitera la visión de confrontación que se viene describiendo en el texto principal del presente artículo, y pregona una expansión de la dinámica defensiva diplomática, comercial y militar entre Estados Unidos y Australia con relación a China en el ámbito del Océano Pacífico a corto y mediano plazos (para un comentario crítico más detallado véase Kevin, 2018). 
B. En contraste con los modelos de presencia y dominación estratégica nacional que mantienen, con resultados mixtos, Estados Unidos, China y Australia en la cuenca y en el Oceáno Pacífico, los países insulares del Pacífico han construido una comunidad de naciones centrada en el desarrollo, la negociación y la seguridad humana colectivos como vías preferenciales para mantener la estabilidad regional y fortalecer sus sistemas de valor cultural y político locales. Una de las fortalezas más interesantes de este sistema es que, al ser colectivo, ha podido mantener una capacidad sumamente eficaz de acción y presión internacional en relación con toda una gama de procesos. El éxito de este modelo se cifra en la Comunidad y en el Foro de Islas del Pacífico como bloques unificados de interés en espacios relevantes como la Asamblea General de las Naciones Unidas, la Unión Europea, y los organismos de financiamiento internacional como el Banco Asiático de Desarrollo y el Banco Mundial (Levine, 2009; Dornan y Newton Cain, 2015; Christie, 2017).

Mientras que el listado de fuerzas armadas estratégicas de las dos principales potencias en Asia-Pacífico es útil para entender la realidad militar "sobre terreno firme", no refleja la naturaleza de la visión estratégica y de influencia geopolítica tal y como existe en las Islas del Pacífico. Esta visión tiene menos que ver con el desarrollo de capacidades bélicas y más con fórmulas de influencia e intercambio laterales.

Lejos de pensar en la suya como una zona de inestabilidad, o bien como un espacio de competencia estratégica entre potencias circundantes, la Comunidad del Pacífico se ha dedicado a atender los problemas más apremiantes que enfrentan sus países miembros. El principal tiene que ver con el cambio climático y la gestión de recursos naturales, sobre todo la pesca en aguas profundas. Es aquí donde las preocupaciones de seguridad regional se transforman, con la visión de los gobiernos isleños, en preocupaciones propias de seguridad humana y desarrollo, sobre todo en relación con el clima y la política ambiental. Por eso, en lugar de colocarlas dentro de la temática de Seguridad, estos retos ofrecen la pauta para el tercer y último subapartado de este artículo (Rich, 2006; Pacific Community, 2015).

\section{CAMBIO CLIMÁTICO Y POLÍTICA AMBIENTAL}

Las naciones insulares del Pacífico se cuentan entre los territorios más vulnerables al cambio climático, esto ha obligado a sus gobiernos, así como a diversos organismos multilaterales entre los que destacan la UNESCO, el Programa de Naciones Unidas para el Medio Ambiente 
(PNUMA), el Programa de Naciones Unidas para el Desarrollo (PNUD) y el Banco Asiático de Desarrollo, además de la CP mediante su Programa para el Medio Ambiente en el Pacífico (SPREP, por sus siglas en inglés), a convertirse en punteros en materia de diplomacia y activismo climático. El éxito de esta actividad se puede medir específicamente en el impacto que las Islas del Pacífico están ejerciendo sobre la manera como se discute y se diseña la política ambiental en los más altos foros internacionales. A su vez, el escenario del Océano Pacífico se ha convertido en una geografía modelo para la forma que toma la investigación científica y social acerca de los efectos del calentamiento global sobre comunidades y naciones (Barnett y Campbell, 2010; Nunn, 2012).

A principios del siglo XXI comenzó a hacerse patente que las Islas del Pacífico representaban una de las llamadas "líneas del frente" del cambio climático; esta percepción se fue formalizando hasta quedar registrada por el Grupo Intergubernamental de Expertos sobre el Cambio Climático (IPCC) de Naciones Unidas, que a partir de su 4o. Informe de Evaluación (IE4) en 2007, señaló la alta vulnerabilidad de las islas pequeñas a los efectos del calentamiento global. En concreto, el IE4 ofreció una síntesis de cinco tipos de transformación socioambiental que afectan de manera desproporcionada a las ecologías y comunidades humanas de Oceanía (Keener, 2012).

El primero es el alza en la temperatura promedio de la atmósfera planetaria, de 0.99 a $3.11^{\circ} \mathrm{C}$ entre 1999 y 2099 , que tendrá un efecto directo en los niveles del mar a raíz del deshielo de glaciares y de los polos de la Tierra. Una segunda transformación medioambiental que se señala en el IE4 de 2007 y que ya se ha registrado desde 2008 en Oceanía es la desestabilización de las zonas de convergencia pluvial intertropicales, que conllevará lluvias más intensas e irregulares (fuera de estación) junto con eventos de sequía e inundación mucho más intensos.

El calentamiento global también generará cambios en los sistemas climáticos regionales, especialmente el conocido como la Oscilación del Sur de El Niño, también conocido como el fenómeno de "El Niño". En concreto, el cambio climático intensifica las temperaturas del aire y de las superficies marítimas, provocando con ello eventos ciclónicos mucho más violentos de lo normal; así, al igual que sucede con los regímenes pluviales, desde hace poco más de una década se han registrado huracanes excepcionalmente violentos en el 
Pacífico insular. Los costos humanos e infraestructurales de estos huracanes han sido altísimos, y el pronóstico en el corto y el mediano plazo es que seguirán ocurriendo.

Otra transformación problemática que se deriva especialmente de los citados cambios en los niveles del mar y en el desequilibrio de los regímenes previos de lluvias y secas estacionales, es que ambos fenómenos están empezando a tener un impacto directo y altamente preocupante, primero sobre la calidad de suelos agrícolas como resultado de la erosión desproporcionada, y segundo, sobre la disponibilidad de fuentes de agua fresca en islas pequeñas. Este segundo fenómeno se refiere a la existencia de pequeños acuíferos o lentes de agua fresca que se forman debajo de islas pequeñas y atolones de tipo calcáreo. Esas reservas locales de agua dependen directamente de la filtración en el subsuelo insular del agua de mar, así como de la renovación que supone el agua pluvial. En un escenario de lluvias y mares desequilibrados, las fuentes de agua fresca de muchísimas poblaciones isleñas vulnerables podrían convertirse en una de las causas más prominentes de migraciones climáticas forzadas.

Finalmente, el IE4 señala la posibilidad de un aumento en el riesgo de brotes epidémicos de enfermedades de cada región, como la malaria, la fiebre del dengue y la ciguatera, a raíz del aumento de la temperatura atmosférica. La asociación entre brotes epidémicos y altas temperaturas está bien documentada en Oceanía y supondría un aumento sensible en la vulnerabilidad de la salud humana de comunidades con acceso limitado a servicios básicos.

Los costos del cambio climático a las economías isleñas del Pacífico están claros. Desde el año 2000 el Banco Mundial estimó que para 2050 los daños por cambio climático podrían sumar decenas de millones de dólares en la infraestructura crítica de las islas, así como en la calidad de vida de sus habitantes. Estos costos no son únicamente cuantitativos, sino que dan lugar a concatenaciones de efectos adversos a lo largo de las industrias clave de las Islas del Pacífico que se mencionaron al principio de este artículo, a saber, el turismo, la pesca y la agricultura. Pero sobre todo el cambio climático supone una amenaza para la vida humana misma en muchas de las islas pequeñas. En este sentido, el costo humano por el calentamiento global se medirá en términos de la capacidad misma de sociedades enteras para sobrevivir en un escenario de recursos primarios degradados, geografías insulares anegadas o inhabitables, y golpes brutales a los sectores productivos y de continuidad cultural de las Islas (Kelman, 2006; Rappaport, 2013). 
La respuesta de la Comunidad del Pacífico no se hizo esperar. A lo largo de la última década varios líderes nacionales de países insulares se han lanzado a la tribuna internacional para llamar la atención acerca de la amenaza que supone el cambio climático para su región. A su vez, los gobiernos de varios países insulares han acelerado la formalización y renovación de estructuras ministeriales y gubernamentales para dar respuesta urgente y coordinada a las transformaciones climáticas, y el puntero en esta esfera ha sido la República de Vanautu, con la creación en 2014 de un Ministerio del Cambio Climático, desde el cual se ha comenzado a organizar un esfuerzo enorme, nacional y regional, de activismo, preparación, investigación y financiamiento con fines de mitigación y adaptación al calentamiento global (Crook y Rudiak-Gould, 2018; Diver, 2018).

Es en el seno de esta actividad colectiva concentrada en una visión de seguridad fincada en la autosuficiencia y el bienestar humanos que se despliega de manera más clara la eficacia del modelo de desarrollo y diplomacia colectiva que caracteriza a las Islas del Pacífico; el ámbito privilegiado de este despliegue es el foro anual de los líderes del Pacífico. Los procesos establecidos y desarrollados en el transcurso del año transcurrido entre los foros de 2017 y 2018 ofrecen un marco ideal para completar la síntesis de actualidades en el Pacífico contemporáneo (Dornan y Newton Cain, 2015; Christie, 2017).

El tema central de la reunión de los líderes del foro en septiembre de 2017 fue "El Pacífico Azul, nuestro mar de islas", concepto derivado de la visión del famoso intelectual y diplomático isleño Epeli Hau'ofa, fallecido hace ya varios años. Durante la reunión de 2017, los líderes del FIP establecieron prioridades regionales enfocadas en la gestión sustentable de los océanos, un renovado activismo y diseño de política ambiental en relación con el cambio climático, un enfoque social en relación con la seguridad regional, y un nuevo marco para la gestión de recursos pesqueros en aguas profundas.

En seguimiento a estas prioridades, durante 2017 los gobiernos del Pacífico promovieron su agenda colectiva del Pacífico Azul en el escenario internacional. Esta labor se vio enormemente asistida y aumentada a raíz de que Fiji ocupó la presidencia de la Asamblea General de las Naciones Unidas para el periodo 2016-2017 —un logro sin precedentes para una nación isleña del Pacífico-. En ese tiempo, el embajador de Fiji, Peter Thomson, utilizó su posición para aumentar la conciencia internacional sobre las preocupaciones regionales. Al final de su gestión en la AGNU, Thomson fue nombrado primer 
Enviado Especial de la ONU para el Océano, un nuevo cargo que emerge directamente de la exitosa labor diplomática del FIP en el contexto internacional.

En 2015 las Naciones Unidas adoptaron diecisiete nuevos Objetivos de Desarrollo Sostenible. Para implementar estos objetivos, Fiji y Suecia organizaron una Conferencia sobre los océanos en la sede principal de las Naciones Unidas, en junio de 2017. Esta conferencia emitió un llamado a la acción relacionado con la degradación ambiental por cambio climático, pero también resultó en un mapa de ruta para atender la creciente amenaza que suponen los desechos plásticos para los mares, así como en la necesidad de generar nuevas políticas de consenso internacional para atender el problema de la pesca ilegal y excesiva en aguas nacionales e internacionales. ${ }^{3}$

\section{CONCLUSIÓN}

Entre 1965 y 1982 la coincidencia de intereses mutuos de las naciones isleñas recién independizadas fue dando lugar a un tipo de regionalismo colectivo en el que el interés común, centrado en el desarrollo humano y la diplomacia mancomunada, se convirtió en la lógica rectora de la arquitectura de seguridad en las Islas del Pacífico. Esta lógica se vincula, en ocasiones de manera virtuosa y en otras contrastada, con el paradigma de desarrollo y seguridad nacional de las potencias grandes y medias continentales con presencia e intereses en el Pacífico. Este segundo paradigma privilegia el concepto realista tradicional de seguridad, basado, tal como el despliegue de fuerzas armadas y una inversión constante, en sistemas de capacidad operativa a distancia, disuasión y defensa de intereses nacionales. El énfasis en este esquema emerge de la idea de seguridad del Estado-nación como fundación de seguridad regional, donde el interés colectivo se mantiene a partir de alianzas políticas y

\footnotetext{
${ }^{3}$ La característica más sorprendente que arroja una aproximación a la gestión de recursos marinos en las Islas del Pacífico es la escasez de información cuantitativa que se posee actualmente para la mayoría de las aguas internacionales de Oceanía, así como de las Zonas Económicas Exclusivas de los países miembros insulares de la Comunidad (Rapapport, 2013: 367). Esta escasez de información, aunada al reto de gestionar la pesca de una veintena de naciones sobre extensiones acuáticas enormes, motivó el establecimiento, en 1979, de la Agencia de Pesca del Foro de Islas del Pacífico, comúnmente conocida como FFA por su abreviación en inglés (Forum Fisheries Agency). La FFA ha seguido un novedoso modelo de gestión que plantea ejes de responsabilidad supranacional para el manejo adecuado del océano más grande del mundo. Asimismo, uno de los objetivos esenciales de la agencia ha sido generar datos que ayuden a saldar la falta de conocimiento que se posee sobre recursos de pesca en el Océano.
} 
militares entre potencias; la seguridad humana y la estabilidad socioeconómica de los países insulares ocupan un escaño secundario en este esquema (Herr, 2015).

Lo anterior pone en evidencia dos problemas importantes: primero, la carencia de una visión integral y positiva acerca del Pacífico, de las potencias medias y globales dominantes (en especial Australia y Estados Unidos), y segundo, la tensión que está generando el choque entre el paradigma estratégico dominante, definido por intereses nacionales estrechos y una definición de supremacía militar sustentada en una visión de suma cero, y aquellas potencias con el paradigma regional de las Islas del Pacífico, que privilegia la seguridad a partir del desarrollo y la estabilidad comunitarios. La gran interrogante que plantea la coyuntura actual es si la República Popular de China podrá superar la tentación de abrirse paso en el Pacífico a partir de procedimientos hegemónicos basados en relaciones económicas asimétricas y coercitivas, o privilegiará la posibilidad de extender su presencia estratégica de manera virtuosa en relación con los países de la Comunidad del Pacífico.

\section{REFERENCIAS BIBLIOGRÁFICAS}

Australian Financial Review, "We Could Work with China in the Region: Scott Morrison". Disponible en: https://www.afr.com/news/we-could-work-with-china-in-the-regionscott-morrison-20181111-h17s3q (consultado el 12 de noviembre de 2018).

Barnett, Jon y John Campbell (2010), Climate Change and Small Island States. Power, Knowledge and the South Pacific, Earthscan, Washington D.C.

Chandler, Jo (2018), "APEC comes to PNG", The Monthly. Disponible en: https://www.themonthly.com.au/issue/2018/november/1540990800/jo-chandler/apeccomes-png (consultado el 10 de noviembre de 2018).

Chang, Charis (2018), "Map Shows Changes in How Much Aid China Provides to the Pacific Region". Disponible en: https://www.news.com.au/world/pacific/map-showschanges-in-how-much-aid-china-provides-to-the-pacific-region/newsstory/351ecd40af458d35c7f44d8c1f7a367f (consultado el 12 de noviembre de 2018). 
Christie, Patrick et al. (2017), "Why People Matter in Ocean Governance: Incorporating Human Dimensions into Large-Scale Marine Protected Areas", Marine Policy, vol. 84, octubre, pp. 273-284.

Crocombe, Ron (2001), The South Pacific, Suva, University of the South Pacific.

Crocombe, Ron (2007), Asia in the Pacific Islands. Replacing the West, Suva, University of the South Pacific.

Crook, Tony y Peter Rudiak-Gould (eds.) (2018), Pacific Climate Cultures. Living Climate Change in Oceania, Berlin, DeGruyter.

Diver, Cameron (2018), "The Ocean in Us: Pacific Regionalism and Identity as a Catalyst for Global Ocean Action", Pacific Community Web Stories. Disponible en: https://www.spc.int/updates/blog/2018/11/the-ocean-in-us-pacific-regionalism-andidentity-as-a-catalyst-for-global (consultado 10 de noviembre de 2018).

Dornan, Matthew (2018), “Australia's Pacific Island Myopia”, The Diplomat, 13 de julio de 2018. Disponible en: https://thediplomat.com/2018/07/australias-pacific-islandmyopia/

Dornan, Matthew (2018), "Labor is Making big Promises for a New Pacific Development Bank, but Many Unanswered Questions Remain", DevPolicyBlog, 31 de octubre de 2018. Disponible en: http://www.devpolicy.org/labor-is-making-big-promises-for-anew-pacific-development-bank-but-many-unanswered-questions-remain-20181031/ (consultado el 10 de noviembre de 2018).

Dornan, Matthew y Tess Newton Cain (2015), "The Moresby Forum: A Reframed Pacific Regionalism?", DevPolicyBlog, 30 de septiembre. Disponible en: http://www.devpolicy.org/the-moresby-forum-a-reframed-pacific-regionalism20150930/ (consultado el 30 de octubre de 2018).

Fish, Tim (2018), "Australia, U.S. set to expand Papua New Guinea naval base", United States Naval Institute, 23 de noviembre. Disponible en: https://news.usni.org/2018/11/23/australia-u-s-set-expand-papa-new-guinea-navalbase (última consulta el 17 de diciembre de 2018). 
Financial Express (2018), “Australia Plans \$1.5b Pacific Fund to Counter China's Influence”. Disponible en: http://thefinancialexpress.com.bd/public/economy/global/australiaplans-15b-pacific-fund-to-counter-chinas-influence-1541662108 (consultado el 9 de noviembre de 2018).

Hasham, Nicole (2018), "Mike Pence announces US-Australia military pact to expand Manus Island naval base", The Sydney Morning Herald, 17 de noviembre. Disponible en: https://www.smh.com.au/politics/federal/mike-pence-announces-us-australiamilitary-pact-to-expand-manus-island-naval-base-20181117-p50goi.html (última consulta el 17 de diciembre de 2018).

Hayward-Jones, Jenny (2018), "Regional Security Dilemma in the Pacific". Disponible en: https://www.lowyinstitute.org/the-interpreter/regional-security-dilemma-pacific (consultado el 12 de noviembre de 2018).

Henderson, John y Greg Watson (2005), Securing a Peaceful Pacific, Christchurch, Canterbury University Press.

Herr, R. A. (2015), "Regional Security Architecture in the Pacific Islands Region: Rummaging through the Blueprints", en A. Rouben y C. Cramer (eds.), Regionalism, Security and Cooperation in Oceania, Honolulu, Asia-Pacific Center for Security Studies, pp. 17-31.

Howes, Stephen (2018), "It Wasn't Meant to be Like this: PNG's Hosting of APEC", East Asia Forum. Disponible en: http://www.eastasiaforum.org/2018/11/11/it-wasnt-meant-tobe-like-this-pngs-hosting-of-apec/ (consultado el 11 de noviembre de 2018).

International Institute for Strategic Studies (2018), Asia-Pacific Regional Security Assessment 2018, publicación electrónica, IISS.

Keener, Victoria W. et al. (2012), Climate Change and Pacific Islands: Indicators and Impacts. Executive Summary of the 2012 Pacific Islands Regional Climate Assessment (PIRCA), Honolulu, East-West Center.

Kelman, Kelman, Ian (2006), "Island Security and Disaster Diplomacy in the Context of Climate Change", Les Cahiers de la Sécurité, vol. 63, pp. 61-94. 
Kevin, Tony (2018), “The diplomatic disaster that was APEC Port Moresby”, citado en el blog Pearls and Irritations del Embajador John Laurence Menaude, https://johnmenadue.com/tony-kevin-the-diplomatic-disaster-that-was-apec-portmoresby/ (última consulta el 17 de diciembre).

Levine, Stephen (2009), Pacific Ways. Government and Politics in the Pacific Islands, Wellington, Victoria University Press.

McGarry, Dan (2018), “A Culture of Corruption”, Vanuatu Daily Post, 15 de septiembre. Disponible en: http://dailypost.vu/news/a-culture-of-corruption/article_446480b3d919-5a54-ae13-53d094605a20.html (consultado el 30 de octubre de 2018).

Moon, Chung-In (2018), "Pacific Islands Forum”, Encyclopaedia Britannica. Disponible en: https://www.britannica.com/topic/Pacific-Islands-Forum. Consultado el 1 de noviembre.

Newton Cain, Tess (2018), Walking the Talk: Is Australia's Engagement with the Pacific a "Step Up" or a Stumble?, Townsville, The Cairns Institute Policy, Paper Series.

Nunn, Patrick D. (2012), Climate Change and Pacific Island Countries. Asia-Pacific Human Development Report Background Papers Series 2012/07, PNUD.

Pacific Community (2015), Pacific Community Strategic Plan 2016-2020. Sustainable Pacific development through science, knowledge and innovation, Nouméa.

Pacific Islands Forum (2018), "Forty-Ninth Pacific Islands Forum: Communiqué", 6 de septiembre. en: https://foreignminister.gov.au/releases/Pages/2018/mp_mr_180906a.aspx (consultado el 30 de octubre de 2018).

Rappaport, Moshe (2013), The Pacific Islands. Environment and Society (Revised edition), Honolulu, University of Hawai Press.

Rich, Ronald et al. (2006), Political Parties in the Pacific Islands, Canberra, Pandanus Books/Research School of Pacific and Asian Studies/Australian National University.

White, Hugh (2010), "Power Shift: Australia's Future Between Washington and Beijing", Quarterly Essay, núm. 39. 